\title{
Susac syndrome following COVID-19 infection
}

\author{
Laura Venditti ${ }^{1,2} \cdot$ Antoine Rousseau $^{3} \cdot$ Claire Ancelet $^{4} \cdot$ Thomas Papo $^{5} \cdot$ Christian Denier $^{1,2}$ (D)
}

Received: 15 October 2020 / Accepted: 10 November 2020 / Published online: 24 November 2020

(c) Belgian Neurological Society 2020

Keywords Susac syndrome $\cdot$ SICRET $\cdot$ COVID-19 $\cdot$ SARS-CoV-2 $\cdot$ Stroke $\cdot$ Vascular endothelial cell

$\begin{array}{ll}\text { Abbreviations } & \\ \text { ACE-2 } & \text { Angiotensin-converting enzyme 2 } \\ \text { ADC } & \text { Apparent diffusion coefficient } \\ \text { COVID-19 } & \text { Coronavirus disease of 2019 } \\ \text { DWI } & \text { Diffusion-weighted imaging } \\ \text { FLAIR } & \text { Fluid-attenuated inversion recovery } \\ \text { MoCa } & \text { Montreal cognitive assessment } \\ \text { PCR } & \text { Polymerase chain reaction } \\ \text { SARS-CoV-2 } & \text { Severe acute respiratory syndrome-coro- } \\ & \text { navirus type 2 }\end{array}$

Dear Editor,

Susac syndrome is a very rare cause of stroke characterized by microvessel occlusion with a typical clinical triad of encephalopathy, branch retinal artery occlusions and sensorineural hearing impairment. Recently, stroke has been described as a possible complication in coronavirus disease of 2019 (COVID-19) patients, with various mechanisms involving inflammation and microvascular events. We report the first case of Susac syndrome occurring after COVID-19 infection.

Laura Venditti

laura.venditti@aphp.fr

1 Department of Neurology, Centre Hospitalo-Universitaire de Bicêtre, Assistance Publique-Hôpitaux de Paris (AP-HP), 78 Rue du Général Leclerc, 94275 Le Kremlin-Bicêtre, France

2 Faculté de Médecine, Université Paris-Saclay, 63 Rue Gabriel Péri, 94270 Le Kremlin-Bicêtre, France

3 Department of Ophtalmology, Centre Hospitalo-Universitaire de Bicêtre, AP-HP, 94275 Le Kremlin-Bicêtre, France

4 Department of Neuroradiology, Centre Hospitalo-Universitaire de Bicêtre, AP-HP, 94275 Le Kremlin-Bicêtre, France

5 Department of Internal Medicine, Centre Hospitalo-Universitaire de Bichat, AP-HP, 75018 Paris, France
A 25-year-old woman was admitted to our stroke unit for acute confusion, transitory right facial palsy and sudden dysarthria. One month prior to admission, she had had fever, flu-like syndrome and anosmia. Although she was not tested with nasopharyngeal polymerase chain reaction (PCR) assay for SARS-CoV-2 (severe acute respiratory syndrome-coronavirus type 2) at this time, infection was later confirmed with serology testing. On stroke unit admission, she had cognitive-especially memory-dysfunction, so severe that the mini-mental state examination (MMSE) and montreal cognitive assessment (MoCa) could not be conducted. Magnetic resonance imaging (MRI) showed multiple acute and subacute ischaemic cortical strokes, in various arterial territories, associated with leptomeningeal enhancement (Fig. 1a-g). A second MRI scan was performed 4 days later because of a worsening of the facial palsy, associated with aphasia and cerebellar ataxia. It showed new strokes, especially in the corpus callosum. Cerebral arteriography was normal. The extensive workup search for usual causes of stroke in young adults was negative. This included HIV, HBV, HCV serology, lupus anticoagulant, anticardiolipin and anti- $\beta 2$-glycoprotein IgG and IgM, anti-nuclear, antiDNA, anti-neutrophil cytoplasmic antibodies, as well as cryoglobulinemia. However, we found the presence of serum anti-endothelial antibodies. Serum C3, C4, and CH50 were normal. The cerebrospinal fluid (CSF) analysis found lymphocytic meningitis with seven elements and high proteinorachia $(1.83 \mathrm{~g} / \mathrm{L})$ without hypoglycorachia and negative microbiological analysis. PCR SARS-CoV-2 in the CSF was negative. Interleukin- 6 and -10 levels were normal in CSF. Full-body computed tomography, positron emission tomography and cardiac echocardiography were normal. Finally, fundus examination showed arteriolar occlusions that were confirmed using fluorescein angiography, which also revealed focal retinal arteriolar vasculitis (Fig. 1h, i), leading to the diagnosis of Susac syndrome. Audiometry was normal. The patient was treated with intravenous pulses of methylprednisolone ( $1 \mathrm{~g}$ per day for 5 days followed by 


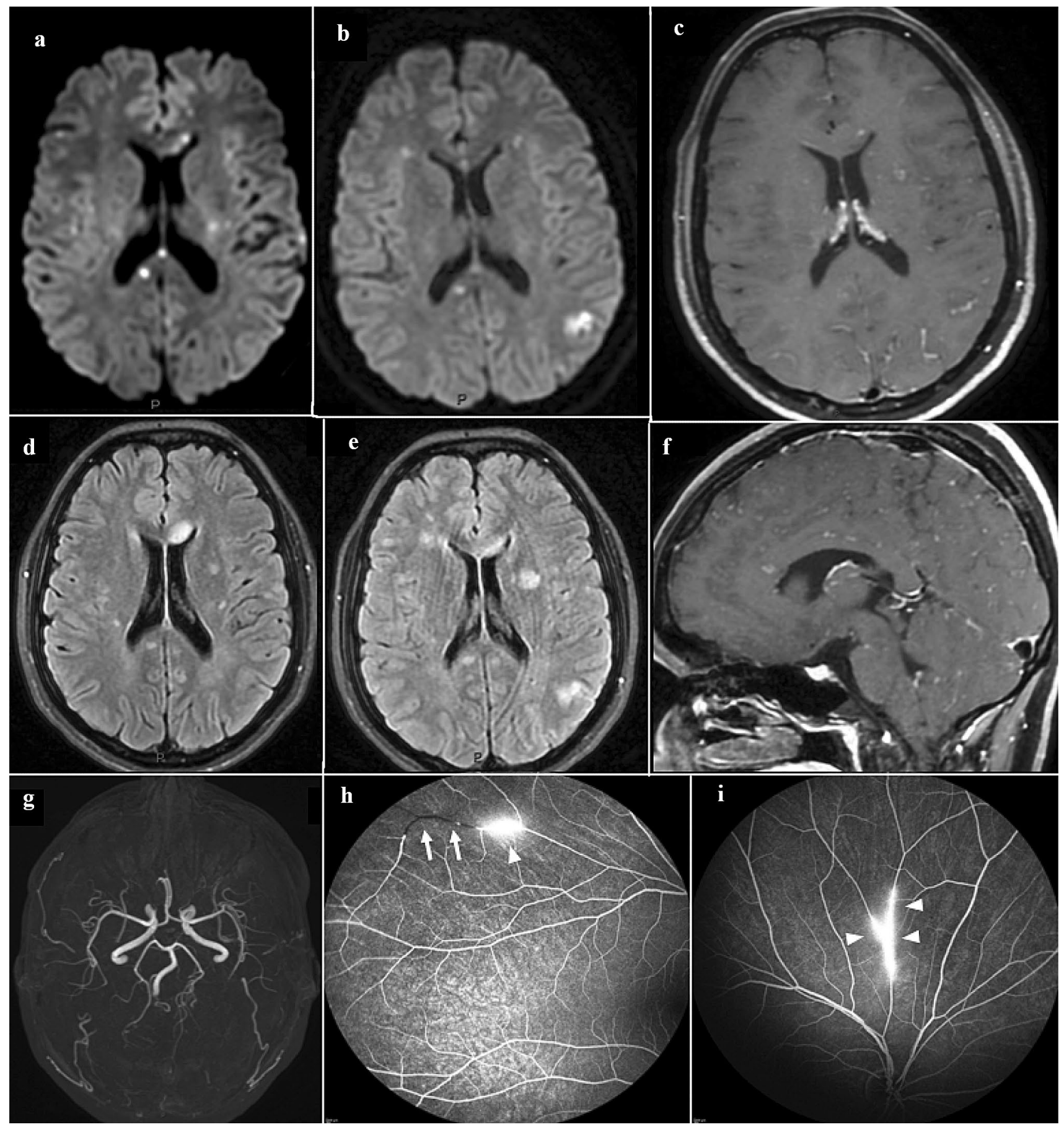

Fig. 1 Cerebral magnetic resonance imaging and fluorescein angiography leading to the diagnosis of Susac syndrome in our patient. Magnetic resonance imaging $\mathbf{a}-\mathbf{g}$ showed multiple acute and subacute infarcts in DWI (a, b) and FLAIR sequences $(\mathbf{d}, \mathbf{e})$, some with restriction of $\mathrm{ADC}$, in various arterial territories (corpus callosum, and

$1 \mathrm{mg} / \mathrm{kg} /$ day of oral prednisone with rapid tapering over 6 weeks). Neurological signs improved quickly with resolution of motor deficit and aphasia. Monthly immunoglobulin therapy was introduced and aspirin was maintained. One right striatum and parietal lobe). It is associated with leptomeningeal and stroke enhancement without vessel enhancement (T1 post gadolinium sequences: $\mathbf{c}, \mathbf{f})$ nor proximal occlusions (TOF reconstruction: g). Right eye late phase fluorescein angiogram $(\mathbf{h}, \mathbf{i})$ shows arteriolar vasculitis (arrowheads) and occlusion (arrows)

month after immunoglobulin infusion, cognitive re-education was stopped because of complete recovery. Cerebral MRI after 2 months showed a decrease in enhanced lesions and no evidence of new ischaemic lesions. 
Neurological manifestations reported in the setting of COVID-19 mainly consist of strokes, seizures, headaches, impaired consciousness or neuro-muscular disorders [1]. Stroke may be explained by hypercoagulopathy, vasculitis and cardiomyopathy [2]. SARS-CoV-2 attaches to angiotensin-converting enzyme 2 (ACE-2) receptor on endothelial cells, and this may lead to endothelial cell dysfunction and trigger a cytokine cascade causing severe coagulopathies and occlusive vasculopathy [2]. Stroke during COVID-19 is usually ascribed to large vessel occlusion. Nonspecific small vessel disease has also been described, for which a mechanism of endothelial dysfunction has been suggested [2,3]. Susac syndrome is a rare cause of stroke with microvessel occlusion occurring mostly in young adults, underdiagnosed, often misdiagnosed and that can lead to severe complications such as deafness, vision loss, dementia and death [4]. Pathophysiology of Susac syndrome remains largely unknown, but is presumed to be mediated by an autoimmune response to an as yet unknown antigen mediated by auto-reactive CD8-positive cells [4]. In our case, the presence of antiendothelial antibodies, which has been previously described in Susac syndrome and underlies the role of endothelial cells in this pathology, co-existed or may have been be enhanced by endothelial dysfunction caused by SARS-CoV-2 infection [4]. Susac syndrome may belong to the spectrum of post-COVID-19 inflammatory vasculopathy [5]. This case has raised the question of a novel mechanism for strokes in COVID-19 patients supported by microvessel occlusions triggered by secondary inflammatory autoimmune disorder, endothelial dysfunctions and/or potential autoimmune endotheliopathy.
Funding None.

\section{Compliance with ethical standards}

Conflict of interest The authors declare that they have no conflict of interest.

Informed consent Obtained.

Consent for publication We obtained written consent for publication from the patient.

\section{References}

1. Helms J, Kremer S, Merdji H et al (2020) Neurologic features in severe SARS-CoV-2 infection. N Engl J Med 382:2268-2270. https://doi.org/10.1056/NEJMc2008597

2. Spence JD, de Freitas GR, Pettigrew LC et al (2020) Mechanisms of stroke in COVID-19. Cerebrovasc Dis. https://doi. org/10.1159/000509581

3. Rothstein A, Oldridge O, Schwennesen H et al (2020) Acute cerebrovascular events in hospitalized COVID-19 patients. Stroke. https://doi.org/10.1161/STROKEAHA.120.030995

4. Magro CM, Poe JC, Lubow M, Susac JO (2011) Susac syndrome: an organ-specific autoimmune endotheliopathy syndrome associated with anti-endothelial cell antibodies. Am J Clin Pathol 136:903-912. https://doi.org/10.1309/AJCPERI7LC4VNFYK

5. Feldstein LR, Rose EB, Horwitz SM et al (2020) Multisystem inflammatory syndrome in U.S. children and adolescents. N Engl J Med 383:334-346. https://doi.org/10.1056/NEJMoa2021680

Publisher's Note Springer Nature remains neutral with regard to jurisdictional claims in published maps and institutional affiliations. 